\title{
Physics potential and prospects at SuperKEKB/Belle II
}

\author{
Bryan Fulsom*广 \\ Pacific Northwest National Laboratory \\ E-mail: bryan. fulsomepnnl.gov
}

The Belle II experiment, being constructed at the KEK laboratory in Japan, is a substantial upgrade of both the Belle detector and the KEKB accelerator. The Belle II experiment aims to record $50 \mathrm{ab}^{-1}$ of data, a factor of 50 more than the Belle experiment. This large data set will be accumulated with low backgrounds and high trigger efficiencies in a clean $e^{+} e^{-}$environment and will provide unprecedented sensitivity to new physics signatures in $B$ and $D$ meson decays as well as $\tau$ lepton decays. This talk will review the physics capabilities of this experiment.

38th International Conference on High Energy Physics

3-10 August 2016

Chicago, USA

* Speaker.

${ }^{\dagger}$ On behalf of the Belle II collaboration. 


\section{Introduction}

The first generation of $B$-Factories, Belle and BaBar, operated over the last decade, producing world-leading measurements of Cabibbo-Kobayashi-Maskawa (CKM) matrix elements and the unitarity triangle, charge-parity violation (CPV) in $B$ meson decays, rare $B$ decays, constraints on New Physics (NP), D mixing, the discovery of exotic/four-quark hadrons, and others [1]. Their discoveries contributed to the awarding of the Nobel prize to Kobayashi and Maskawa in 2008. The Belle II experiment builds upon these successes. It is designed to be the next generation $B$-Factory, with a goal of searching for NP via precision measurements. The focus of this talk is a description of the physics prospects of this experiment.

Belle II has some specific advantages in the era of the LHC. It will be sensitive to NP mass ranges above direct production, competing in mass reach with the LHC experiments in certain scenarios. The "clean" experimental environment of $e^{+} e^{-}$collisions allows for greater reconstruction and flavor-tagging capability, and has advantages for $\tau$ decays and final states including neutral particles (e.g. $\gamma, \pi^{0}, K_{L}, v$, etc.). The ultimate goal of the experiment is to collect a dataset approximately 50 times larger than that of the previous generation $B$-Factories.

To achieve this, the KEKB accelerator is being upgraded to "SuperKEKB". The upgraded accelerator aims to have a forty times greater instantaneous luminosity by squeezing the $e^{+} e^{-}$ beams to nanometer size, and by increasing the current by a factor of two [2]. The milestone of first circulation of electrons and positrons in the machine was recently demonstrated in February 2016.

To cope with the increased level of background, radiation damage, and event rates from the order of magnitude increase in luminosity, the Belle detector is being rebuilt as "Belle II" [3]. This upgrade includes a new vertex detector composed of silicon pixels and strips, an expanded small-cell drift chamber for tracking, a new particle identification system based on Cherenkov light time-of-propagation in the barrel and focusing ring-imaging in the endcap, upgrades to the electromagnetic calorimeter readout, and replacement of endcap and inner barrel muon detector layers with plastic scintillator. There are also necessary updates to the trigger, data acquisition, and computing capabilities.

The Belle II collaboration currently consists of $\sim 700$ members from $\sim 100$ institutions in 23 countries. Operations began with an accelerator commissioning run called "Phase 1" in early 2016. The next step, "Phase 2", is planned for the fall of 2017. This period will include the Belle II detector without the vertex detectors, and will see first $e^{+} e^{-}$collisions, studies of beam backgrounds, and potentially the first physics output. "Phase 3" will be the start of the nominal running period with the full detector, scheduled for the end of 2018. Belle II is expected to operate into the next decade, collecting at least $50 \mathrm{ab}^{-1}$ of data (compared to $\sim 1.5 \mathrm{ab}^{-1}$ from Belle and BaBar combined).

\section{Physics Topics of Interest}

This section highlights some of the potential topics of interest to be studied at Belle II. Though the Belle II physics program will be wide-ranging, this talk particularly covers items related to physics beyond the Standard Model (SM), such as lepton flavor violation and contributions from 
new particles entering in loop decays, and potential results from the early operating period planned for next year.

\subsection{Leptonic $B$ decays}

The leptonic $B$ decay $B \rightarrow \tau \nu$ is potentially sensitive to NP contributions from charged Higgs bosons coupling to the $B$ meson and $\tau$ and $v_{\tau}$. This could lead to an enhancement in the branching fraction over the $W$-mediated SM prediction of $(1.11 \pm 0.28) \times 10^{-4}$ [4]. The current world average from the CKMfitter group is $\left(0.848_{-0.055}^{+0.036}\right) \times 10^{-4}$ [4]. Belle II at $50 \mathrm{ab}^{-1}$ aims to reduce the uncertainty on this measurement to less than $5 \%$ in a single measurement, approaching the level of sensitivity from theory.

\subsection{Semileptonic $B$ decays}

The decay $B \rightarrow D^{*} \tau \nu$ is similarly sensitive to charged Higgs contributions. Compared to $B \rightarrow \tau \nu$, there is a larger expected branching fraction, and less theoretical uncertainty by exploiting cancellations in ratios of decays to $\tau$ final states compared to $e$ and $\mu$, and $D$ and $D^{*}$. This is a topic of intense interest due to recent results from BaBar, Belle, and LHCb [5] that, when combined, show a deviation of greater than $3 \sigma$ from the SM expectation. The current uncertainties associated with the $B$-Factory measurements are $16(9) \%$ for the $D^{(*)} \tau \nu_{\tau}$ final state. At the full target luminosity Belle II is expected to reduce the uncertainty to $2-3 \%$, which would represent a significant deviation from the SM if the current world average holds.

\section{$2.3 b \rightarrow s$ decays}

Radiative penguin decays are another topic of interest due to existing hints for physics beyond the SM via new particles entering into decay loop diagrams. Measurements of the forwardbackward asymmetry in $b \rightarrow s \ell \ell$ transitions at low $q^{2}$ ( $\ell \ell$ invariant mass squared) from LHCb and Belle [6] are in tension with SM predictions. While LHCb is expected to dominate $B \rightarrow K^{*} \mu^{+} \mu^{-}$ measurements, Belle II can contribute in a complementary way by measuring the $\tau^{+} \tau^{-}$and $e^{+} e^{-}$ final states, and inclusive $X_{s}$ modes (i.e. by summing many exclusive final states). Additionally, Belle II will be uniquely sensitive to $B \rightarrow K^{(*) 0} v \bar{v}$. SM predictions for this decay are in the range of $10^{-6}$ [7], with current Belle limits at $10^{-5}$ [8]. Belle II should be able to measure these decays at the SM rate with an uncertainty of $\sim 20 \%$. Belle II will also contribute by testing NP models via precise measurements of $b \rightarrow d \gamma$ and $b \rightarrow s \gamma$ rates and charge-parity asymmetry.

\subsection{NP in CPV, CKM, and charm}

$\mathrm{CPV}$ and CKM measurements that comprised the foundation of the experimental program at the $B$-Factories can be extended in Belle II to probe for NP. The sum of the angles of the unitarity triangle is currently $\alpha+\beta+\gamma=175^{\circ} \pm 9^{\circ}$ [4]. The combined reach of many relevant decay modes at Belle II will reduce the individual angular uncertainties to $\delta \alpha \sim 1^{\circ}, \delta \beta \sim 0.3^{\circ}$, and $\delta \gamma \sim 1.5^{\circ}$, which will test the closure condition, and hence NP contributions, to an unprecedented level. Measurement of the $\sin (2 \beta)$ parameter from the $B \rightarrow J \psi K_{S}^{0}$ "golden mode" in $\bar{b} \rightarrow c \overline{c s}$ decays can be compared to that from $\bar{b} \rightarrow s \overline{s s}$ decays $\left(\right.$ e.g. $\left.B \rightarrow\left(\eta^{\prime}, \phi\right) K\right)$. The current $\sin (2 \beta)$ values are $+0.682 \pm 0.019$ and $+0.64 \pm 0.03$ respectively, showing some potential hint of a deviation that 
could arise from additional particles entering into loop decays for the latter. Theoretical uncertainties from the SM are at the level of $\sim 1 \%$ [9], while Belle II should be able to challenge these with an ultimate experimental precision of $\sim 1.2 \%$. The charm sector also offers a wealth of potential topics, given the large cross section for charm production at the planned Belle II operating energies. This includes reductions in the uncertainties on $D^{0} \bar{D}^{0}$ mixing parameters, and the search for CPV and rare decays.

\subsection{Lepton flavor violation (LFV)}

Searches for LFV, particularly in the $\tau$ sector, will be particularly advantageous for Belle II. $\tau^{+} \tau^{-}$pairs are copiously produced in $e^{+} e^{-}$collisions at the Belle II operating energy, and the topology of the decay can be exploited to reconstruct events with missing neutrinos from $\tau$ decay. The LFV rate is predicted to be vanishingly small in the SM (e.g. $\left.10^{-25}\right)$, but NP models could potentially enhance these decay rates to $10^{-7}-10^{-10}$, which are in reach of Belle II. Belle II is expected to challenge NP models by improving the upper limits on dozens of lepton flavor and baryon number violating modes, from current limits of $10^{-7}$ by at least an order of magnitude or better, to the range of $10^{-9}$ [9].

\subsection{NP with low multiplicity}

The low-multiplicity environment of $e^{+} e^{-}$decays will allow Belle II to search for NP in the "dark sector" (where a "dark photon" $A^{\prime}$ can mix with the SM photon and decay to fermions or other non-interacting particles, " $\chi$ ”) or with light Higgs boson candidates [10]. One specific example is to search for $e^{+} e^{-} \rightarrow \gamma A^{\prime}(\chi \chi)$, which amounts to triggering on a single (monoenergetic) photon. Development of specialized single photon triggers is underway to establish a sensitivity to these decays that was largely unavailable at the $B$-Factories. Belle II will be the only way to explore large areas of the parameter space of $A^{\prime}$ mass and the dark coupling to the $\mathrm{SM}, \varepsilon$ [11]. It may also be possible at Belle II to probe NP in other invisible decays, e.g. $\Upsilon(1 S) \rightarrow \chi \chi$ by first tagging and triggering on $\Upsilon(m S) \rightarrow \pi^{+} \pi^{-} \Upsilon(1 S)$ decays.

\subsection{Quarkonium and early physics prospects}

Because it will take a few years to accumulate a data sample to sufficiently improve upon existing $B$-Factory results, there may be an opportunity to have an earlier physics impact by operating at an alternate center-of-mass energy during the initial "Phase 2" and "Phase 3" of the experiment. The Belle II collaboration is considering where collection of a limited data set of (10-100) $\mathrm{fb}^{-1}$ would offer the greatest return. Below the $\Upsilon(4 S)$ energy, Belle II could run at $\Upsilon(2 S)$ or $\Upsilon(3 S)$ energies to access the bottomonium and dark sectors. Another possibility could be to scan for the direct production of yet-to-be-discovered $D$-wave $\Upsilon$ states (with subsequent decay to the predicted $F$ wave level of bottomonium). Energies above $\Upsilon(4 S)$ are attractive for the study of exotic four-quark states. One proposal would be to extend Belle studies of the $\Upsilon(6 S)$ energy region analogous to those that led to the discovery of the $h_{b}(2 P)$ bottomonium state and charged $Z_{b}^{ \pm}(106 X X)$ mesonic "molecules" [12]. While the final decision to operate at energies other than $\Upsilon(4 S)$ will depend on many factors, such as accelerator performance and detector comissioning, and will be made at a later time, preparations for this type of early operating scenario have begun. In any case, Belle II 
will continue to lead the study of quarkonium and exotic $X Y Z$ states at all phases and energies of the experiment, as Belle has done [13].

\section{Summary}

The SuperKEKB and Belle II experiment upgrades are well underway. The acclerator achieved first circulation of $e^{+} e^{-}$beams in February 2016. Accelerator and detector commissioning is expected to continue during a dedicated run period in Fall 2017, with the nominal start of the experiment in late 2018. Belle II represents the next generation flavor factory, expected to collect at least fifty times as much data as its predecessors, with improved detector capabilities. The experimental environment offers sensitivity to new physics and final states with neutral particles that is both complementary to and competitive with experiments at the LHC. The main goal of Belle II is to search for new physics via high-statistics precision measurements. It will offer a wide-ranging physics program, covering charge-parity violation, (semi-)leptonic penguin decays, lepton flavor violation, the dark sector, exotic hadrons, and many other topics through the coming decade.

\section{References}

[1] A.J. Bevan, et al., The Physics of the B Factories, Eur. Phys. J. C74, 3026 (2005).

[2] SuperKEKB Design Report (2014), https://kds.kek.jp/indico/event/15914/.

[3] T. Abe, et al., Belle II Technical Design Report, KEK Report 2010-1 (2010).

[4] J. Charles, et al. (CKMfitter Group), Eur. Phys. J. C41, 1 (2005), updated results and plots available at: http://ckmfitter.in2p3.fr.

[5] World average values here include prelimary results from Belle shown at this conference, derived by P. Urquijo, private communication (2016).

[6] Y. Sato, et al. (Belle Collaboration), Measurement of the lepton forward-backward asymmetry in $B \rightarrow X_{s} \ell^{+} \ell^{-}$decays with a sum of exclusive modes, Phys. Rev. D93, 032008 (2016).

[7] A.J. Buras, et al., $B \rightarrow K^{(*)} v \bar{v}$ decays in the Standard Model and beyond, J. High Energy Phys. 02, 184 (2015).

[8] O. Lutz, et al. (Belle Collaboration), Search for $B \rightarrow h^{(*)} v \bar{v}$ with the full Belle $\Upsilon(4 S)$ data sample, Phys. Rev. D87, 111103 (2013).

[9] Y. Amhis, et al. (Heavy Flavor Averaging Group), Averages of b-hadron, c-hadron, and $\tau$-lepton properties as of summer 2014 [arXiv:1412 . 7515], and online update at http://www.slac.stanford.edu/xorg/hfag.

[10] See for example: R. Essig, et al., Dark sectors and new, light, weakly-coupled particles, [arXiv:1311.0029].

[11] $\varepsilon-m_{A^{\prime}}$ exclusion plots courtesy of C. Hearty, private communication.

[12] R. Mizuk, et al. (Belle Collaboration), Energy scan of the $e^{+} e^{-} \rightarrow h_{b}(n P) \pi^{+} \pi^{-}(n=1,2)$ cross sections and evidence for $\Upsilon(11020)$ decays into charged bottomonium-like states, [arXiv:1508.06562]. 
[13] See for example: S.-K. Choi, et al. (Belle Collaboration), Observation of a narrow charmoniumlike state in exclusive $B^{ \pm} \rightarrow K^{ \pm} \pi^{+} \pi^{-} J / \psi$ decays, Phys. Rev. Lett. 91, 262001 (2003); A. Bondar, et al. (Belle Collaboration), Observation of two charged bottomoniumlike resonances in $\Upsilon(5 S)$ decays, Phys. Rev. Lett. 108, 122001 (2012). 